\title{
RESULTS AND PROGNOSTIC FACTORS IN RESECTIONS OF PRIMARY TRACHEAL TUMORS: A MULTICENTER RETROSPECTIVE STUDY
}

\author{
J. F. Regnard, $\mathrm{MD}^{\S}$ \\ P. Fourquier, $\mathrm{MD}^{\S}$ \\ P. Levasseur, $\mathrm{MD}^{\S}$ \\ The French Society of \\ Cardiovascular Surgery \\ Sponsor: \\ P. Pairolero, MD
}

To determine long-term survival and prognostic factors, 208 patients with primary tracheal tumors were evaluated in a retrospective multicenter study including 26 centers. Ninety-four patients had squamous cell carcinoma, four had adenocarcinoma, 65 had adenoid cystic carcinoma, and 45 patients had miscellaneous tumors. The following resections were performed: tracheal resection with primary anastomosis, 165; carinal resection, 24; and laryngotracheal resection, 19. Postoperative mortality rate was $10.5 \%$ and correlated with the length of the resection, the need for a laryngeal release, the type of resection, and the histologic type of the cancer. Fifty-nine percent of patients with tracheal cancer and $43 \%$ of patients with adenoid cystic carcinomas had postoperative radiotherapy. The 5- and 10-year survivals, respectively, were $73 \%$ and $57 \%$ for adenoid cystic carcinomas and $47 \%$ and $36 \%$ for tracheal cancers $(p<0.05)$. Among patients with tracheal cancers, survival was significantly longer for those with complete resections than for those with incomplete resections. On the other hand, the presence of positive lymph nodes did not seem to decrease survival. Postoperative radiotherapy increased survival only in the case of incompletely resected tracheal cancers. Long-term prognosis was worsened by the occurence of second primary malignancies in patients with tracheal cancers and by the occurence of late pulmonary metastases in patients with adenoid cystic carcinomas. (J THORAC CARDIOVASC Surg 1996;111:808-14)
$\mathrm{P}$ rimary tracheal tumors, which are rare and rarely resectable, represent a heterogeneous group of benign and malignant tumors. Few large series have been reported. ${ }^{1-5}$ Surgical resection is considered the treatment of choice for such tumors when they are limited and resectable. Prognosis and indications for adjuvant treatment, however, remain unclear. In light of these facts, we decided to make a retrospective multicenter study to examine a large number of cases of resection to determine long-term survival, prognostic factors, and indication of adjuvant radiotherapy.

For the French Society of Thoracic and Cardiovascular Surgery (see Appendix for authors participating in study).

Read at the Seventy-fifth Annual Meeting of The American Association for Thoracic Surgery, Boston, Mass., April 23-26, 1995.

Address for reprints: Jean-François Regnard, MD, Hôpital Marie Lannelongue, 133 Ave. de la Résistance, 92350 Le PlessisRobinson, France.

${ }^{\circledR}$ By invitation.

Copyright (C) 1996 by Mosby-Year Book, Inc.

$0022-5223 / 96 \$ 5.00+0 \quad \mathbf{1 2 / 6 / 7 0 5 9 8}$

\section{Patients and methods}

Patient characteristics. A questionnaire was sent to all members of the French Society of Thoracic and Cardiovascular Surgery to collect data on all patients operated on during a 23-year period. Data was collected from 26 responding surgical centres (see Appendix) on 208 patients operated on between 1970 and 1993. Overall, only 10 teams operated on 10 patients or more.

Ninety-eight patients had a tracheal cancer (94 squamous cell cancers and four adenocarcinomas), 65 patients had an adenoid cystic carcinoma, and 45 patients had another tracheal tumor. The last group represented a multitude of tumor types and varying degrees of malignancy, including both epithelial and mesenchymal neoplasms (Table I).

There were 148 male and 60 female patients; ages averaged 50 years and ranged from 8 to 75 years. Tracheal cancers occurred predominantly in men (92.5\%) with a mean age of 54 years; most were cigarette smokers. On the other hand, adenoid cystic carcinomas occurred in patients with a mean age of 48 years, with a male-female ratio near 1 ( $54 \%$ female).

Clinical symptoms were generally related to the tracheal obstruction (dyspnea, 42\%; and acute respiratory failure, $29 \%$ ); in contrast, some patients had nonspecific tracheobronchial symptoms (cough, 30\%; and hemoptysis, $36 \%$ ). The mean duration of symptoms was 4 months for tracheal cancers, 8 months for the group with assorted 
Table I. Histology of resected tracheal tumors

\begin{tabular}{llr}
\hline & \multicolumn{1}{c}{ Tumor type } & $n$ \\
\hline Epithelial tumor & & \\
Malignant & Squamous cell carcinoma & 94 \\
& Adenocarcinoma & 4 \\
Low grade & Adenoid cystic carcinoma & 65 \\
& Mucoepidermoid tumor & 5 \\
Carcinoid & 9 \\
& Papilloma & 2 \\
Menign & Adenoma & 3 \\
Benchign & Myoepithelial cell tumor & 1 \\
& & \\
& Neurofibroma & 2 \\
& Lipoma & 1 \\
& Fibroma & 2 \\
& Abrikosoff tumor & 3 \\
& Chondroma & 5 \\
& Leiomyoma & 5 \\
& Schwannoma & 1 \\
& Plasmocytoma & 2 \\
& Miscellaneous & 2 \\
Malignant & Chondrosarcoma & 1 \\
& Melanoma & 1 \\
\hline
\end{tabular}

benign and malignant tumors, and 12 months for adenoid cystic carcinomas. Tumors were located on the subglottic trachea in 19 patients, on the cervical or mid trachea in 163 patients, and on the carinal area in 26 patients.

Seventy-one patients underwent a first procedure of relief of tracheal obstruction to treat an acute respiratory failure or better define location and size of the tumor and to allow better respiratory preparation before surgical resection. The tumor was initially removed by the following methods: 62 patients underwent a laser treatment, five patients underwent bronchoscopic relief of airway obstruction and two underwent cryotherapy. Two who were patients operated on at the beginning of the series underwent tracheotomy as a primary procedure.

Surgical approach was made through a cervicotomy in 61 patients, cervicotomy with split sternotomy in 23 patients, full median sternotomy in 75 patients, and right lateral thoracotomy in 41 patients. Eight patients had complex surgical approaches, such as trap door incision.

One hundred forty-six patients underwent circumferential tracheal resection with primary reconstruction, 19 patients underwent partial tracheal resection (wedge resection), 24 underwent carinal resection, and 19 underwent laryngotracheal resection (seven associated laryngectomies and 12 partial cricoid resections according to the Pearson technique ${ }^{6}$ ). Intraoperative ventilation was performed through the operative field with canulation of the distal trachea in 116 patients, 54 patients had conventional ventilation with a small tracheal tube left in place during the resection, and 38 patients had high-frequency jet ventilation.

Tracheal resection had to be extended to the thyroid in 30 patients, to the esophagus in four patients (lateral removal of the esophagus wall), to one lung in four patients (extensive invasion of one main bronchus), to a recurrent laryngeal nerve in seven patients, and to the
Table II. Specimen characteristics

\begin{tabular}{lccc}
\hline & $\begin{array}{c}\text { Tracheal } \\
\text { cancers } \\
(n=98)\end{array}$ & $\begin{array}{c}\text { Adenoid } \\
\text { cystic } \\
(n=65)\end{array}$ & $\begin{array}{c}\text { Other } \\
(n=45)\end{array}$ \\
\hline $\begin{array}{l}\text { Length of resection (mm) } \\
\text { Positive resection margins }\end{array}$ & $\begin{array}{c}(n+5 \pm 14 \\
\quad \%)\end{array}$ & $38.5 \pm 14$ & $29 \pm 13$ \\
Positive lymph node & 30 & 6 & 9 \\
Complete resection & 70 & 59 & 0 \\
Incomplete resection & 30 & 41 & 91 \\
\hline
\end{tabular}

vena cava in two patients. In addition to systematic anterior tracheal dissection and cervical flexion, 63 patients underwent laryngeal release to lessen tension on the anastomosis. Fifty-one patients underwent a Dedo-Fishman procedure, ${ }^{7}$ nine patients underwent a Montgomery procedure, ${ }^{8}$ and three patients underwent both.

Table II shows specimen characteristics according to the three histologic types. Most patients with positive resection margins had only microscopic tumors present at the resection margins.

Methods. This series was a retrospective multicenter study. Follow-up data were obtained for all but 10 patients until death or completion of the study. Mean follow-up was 4 years for patients operated on for tracheal cancers and 6 years for patients operated on for adenoid cystic carcinomas or other tumors.

Survival was calculated by the actuarial method, ${ }^{9}$ including postoperative deaths. Statistical comparisons between survival curves were performed by the log rank test. The difference was considered significant when the $p$ value was 0.05 or less.

\section{Results}

Postoperative course. One hundred twenty-four patients had uneventful postoperative courses; in contrast, 84 patients $(41 \%)$ had postoperative complications (anastomotic leakage, 25; pneumopathy, 13; and aspiration on deglutition, 29). Twenty-two patients $(10.5 \%)$ died after operation. Four factors were significantly associated with postoperative complications: the length of resection $(p<0.001$; the longer the tracheal resection, the higher the morbidity), the need for laryngeal release $(p<$ 0.001 ), the type of surgical procedure (laryngotracheal or carinal resection vs standard tracheal resection; $p<0.001$ ), and the histologic type (postoperative mortality rate was three times higher for squamous cell carcinomas than for other tumors $(p<0.05)$. The older age of the patient, positive margins at resection, and lymph node involvement did not significantly increase the postoperative complication rate. 


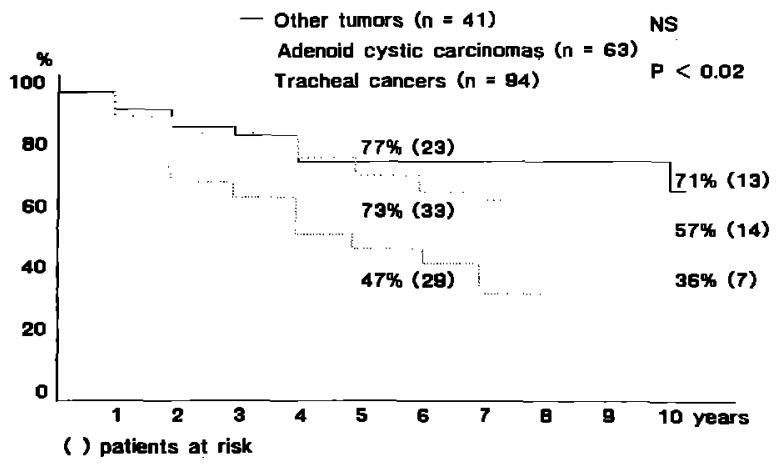

Fig. 1. Actuarial survival curves according to histologic type (excluding 10 patients unavailable for follow-up). NS, Not significant.

Table III shows the adjuvant treatment given after resection according to the two main histologic types. Postoperative treatment was not carried out per protocol in this retrospective study. Adjuvant treatment was administered more frequently to patients with incomplete resection or positive lymph nodes: $31 \%$ in patients with complete resections versus $71 \%$ in those with incomplete resections, and $35 \%$ in patients with negative nodes versus $80 \%$ in those with positive nodes.

Long-term functional results were excellent in 137 patients $(76 \%)$. Eight $(4 \%)$ patients had recurrent granulomas, nine patients had an asymptomatic stenosis, and 26 patients $(14 \%)$ had a symptomatic stenosis. Late complications necessitated laser treatment in 16 patients, tracheal stents in 12 patients, reresection in three patients, and permanent tracheotomy in four patients. Late complications (granulomas and stenosis) were significantly more frequent among patients who had postoperative locoregional complications (tracheal dehiscence and mediastinitis) than among those who had an uneventful postoperative course. In fact, in absence of locoregional complication, $86 \%$ of the patients had excellent long-term functional results, and only $8 \%$ had a symptomatic stenosis. In cases of postoperative locoregional complication, only $56 \%$ of the patients had excellent long-term results, and $27 \%$ had a residual symptomatic stenosis $(p<0.001)$.

Oncologic results. Oncologic results were mainly analyzed with respect to the two main histologic types (tracheal cancers and adenoid cystic carcinomas). Among the patients operated on for tracheal cancers, 23 patients had locoregional recurrences and 10 had distant metastases, with a mean diseasefree interval of 18 months. Furthermore, 17 patients
Table III. Adjuvant treatment (patients surviving to resection)

\begin{tabular}{lcc}
\hline & $\begin{array}{c}\text { Tracheal } \\
\text { cancers } \\
(n=83)\end{array}$ & $\begin{array}{c}\text { Adenoid cystic } \\
\text { carcinomas } \\
(n=60)\end{array}$ \\
\hline None & 34 & 34 \\
Radiotherapy & 44 & 26 \\
Radiotherapy + chemotherapy & 5 & 0 \\
\hline
\end{tabular}

had a second primary cancer (lung, 11; tongue, four; esophagus, one; and miscellaneous, one). In contrast, among patients operated on for adenoid cystic carcinomas, 12 had distant metastases (pulmonary metastases, 12; associated with local recurrence, four; pleural metastases, one; and bone metastases, one), and nine patients had a locoregional recurrence. Recurrences occurred later for adenoid cystic carcinomas (mean interval 51 months) than for tracheal cancers (mean interval 18 months; $p<$ 0.01 ).

Fig. 1 shows the actuarial survival curves according to histologic type. As might be expected, prognosis for the heterogeneous group of benign and low malignant tumors was good, with 5- and 10-year survivals of $77 \%$ and $71 \%$, respectively. Survival was significantly longer for patients with adenoid cystic carcinomas than for patients with tracheal cancers: 5- and 10-year survivals were respectively, $73 \%$ and $57 \%$, and $47 \%$ and $36 \%$ (log rank test, $p<0.05$; Fig. 1). Among patients with tracheal cancers, prognosis was significantly better for patients who had a complete resection than for those who had an incomplete resection; in fact, 5-year survivals were, respectively, $55 \%$ and $25 \%(p<0.02)$.

The presence of positive versus negative lymph nodes did not significantly decrease survival (Fig. 2), but $80 \%$ of patients with positive nodes underwent postoperative radiotherapy, versus $46 \%$ of patients with negative nodes $(p<0.001)$. Postoperative radiotherapy did not significantly improve survival for patients with completely resected tracheal cancers (Fig. 3), however; postoperative radiotherapy improved survival significantly only in patients with incomplete resection (Fig. 4). Similarly, postoperative radiotherapy did not significantly improve survival for patients with either positive or negative lymph nodes.

Among patients with adenoid cystic carcinomas, survival seemed better for patients with complete resection than for those with incomplete resection, but the difference did not reach a significant level 


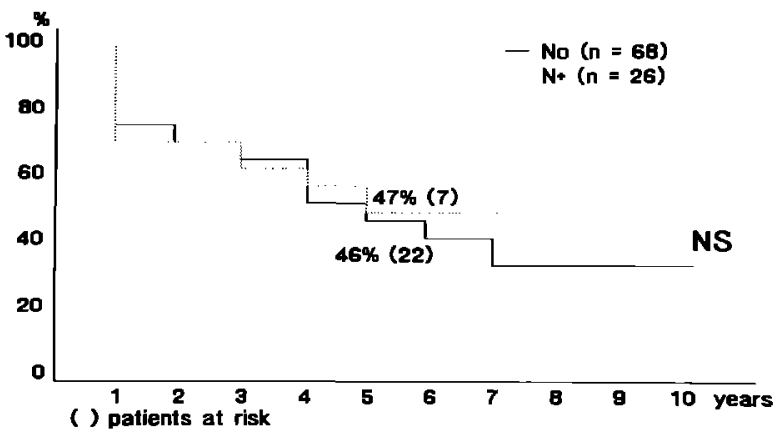

Fig. 2. Survival according to lymph node involvement in tracheal cancers. NS, Not significant.

$(p<0.20$; Fig. 5). Similarly, postoperative radiotherapy or radiochemotherapy did not improve survival in the case of either complete or incomplete resection.

\section{Discussion}

Primary tracheal tumors are rare, and few large series have been reported. A prolonged period is needed to accumulate a sufficient number of cases, so we decided to make a retrospective multicenter study among the members of the French Society of Thoracic and Cardiovascular Surgery. As a result, this study represents one of the largest series reported in the literature, ${ }^{1-4}$ especially considering the number of squamous cell tracheal carcinomas. In contrast with the previously reported series, this series is characterized by the use of the actuarial method and the log rank test to better define long-term prognosis after resection and prognostic factors. Because of the retrospective and multicenter aspect of the series, however, surgical techniques, postoperative results, and indications for adjuvant treatment were heterogeneous.

Oncologic results. This study confirms the good results of surgical resection of primary tracheal tumors. Long-term results in particular seem better for resectable squamous cell tracheal cancers than for resectable lung cancers. ${ }^{3}$ Surgical resection appears to be the treatment of choice for such tumors. ${ }^{1,2}$

According to Grillo and Mathisen, ${ }^{1}$ irradiation alone does not appear to be an appropriate treatment for tracheal cancers. They compared the outcomes of patients operated on with those who were treated by irradiation alone and found that among the 17 patients with squamous cell tracheal cancers only one patient treated by irradiation alone was alive 1 year after the treatment; in contrast, 18 of the

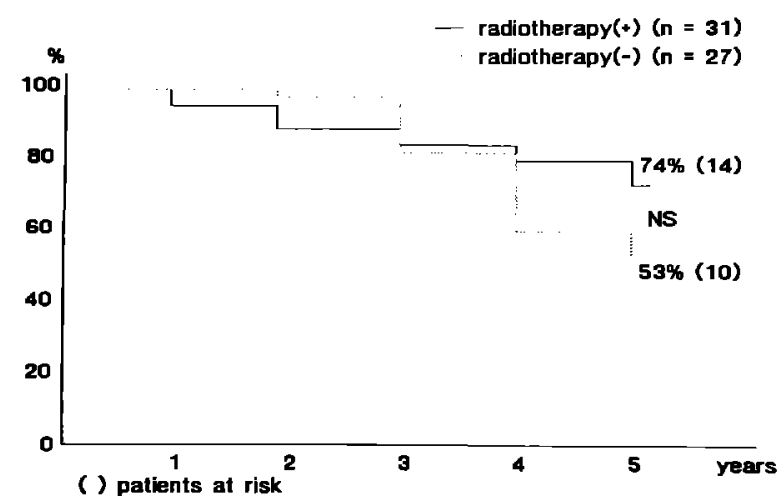

Fig. 3. Influence of adjuvant radiotherapy on survival of patients with completely resected tracheal cancers (excluding patients unavailable for follow-up and postoperative deaths). NS, Not significant.

27 patients who underwent resection were still alive. Similar results were observed in adenoid cystic carcinomas. ${ }^{1}$ Most authors recommend that irradiation alone should be reserved for patients with nonresectable disease. ${ }^{1,10,11}$

In our series, the best prognostic factor for tracheal cancers was the completeness of resection; survival was significantly better among patients with complete resections than among those with incomplete resections. Incomplete resection was generally related to positive resection margins; this result emphasizes the necessity of frozen-section analysis during operation to ensure performance of complete resection. Most patients with positive resection margins had only microscopic tumor residues found in the specimen, however; as a result, some patients with positive resection margins had long-term survival when adjuvant radiotherapy was performed (Fig. 4). Although complete resection should be attempted, in some instances it is impossible to extend the resection without endangering the integrity of the anastomosis by creating excessive anastomotic tension. As a result, the surgeon must sometimes compromise total resection for safety.

The rate of positive resection margins in this series was quite high; in fact, some surgical teams did not use systematic frozen-section analysis. In the series of Grillo and Mathisen, ${ }^{1}$ frozen-section analysis was systematically performed during the operation; nevertheless, the positive resection margin rates in that series were also quite high $(23 \%$ for tracheal cancers and $29 \%$ for adenoid cystic carcinomas). These results confirm the difficulty of per- 


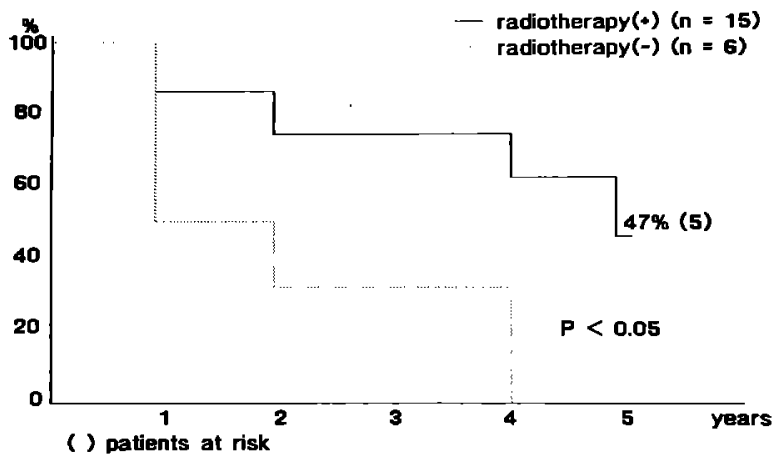

Fig. 4. Influence of adjuvant radiotherapy on survival of patients with incompletely resected tracheal cancers (excluding patients unavailable for follow-up and postoperative deaths).

forming complete resection of tracheal tumors without compromising the integrity of the procedure.

Lymph node involvement did not seem to influence long-term outcome in this series. In contrast to the series of Grillo and Mathisen, ${ }^{1}$ patients with positive lymph nodes had a similar outcome to those without lymph node involvement (Fig. 2). We should point out, however, that $80 \%$ of the patients with positive lymph nodes received postoperative irradiation, compared with $35 \%$ of those with negative lymph nodes. In our series postoperative radiotherapy did not significantly improve survival in patients with either negative or positive nodes. Nevertheless, postoperative radiotherapy significantly improved survival among patients with incomplete resection, generally related to positive resection margins. Because this was not a randomized study and indications for postoperative radiotherapy varied from one team to another, it is not possible to draw definite conclusions from this series. We therefore agree with Grillo and Mathisen, ${ }^{1}$ who emphasize the narrow margins often obtainable in tracheal resections and recommend postoperative radiotherapy for all patients, even when the margins and lymph nodes are histologically negative.

The prognosis for adenoid cystic carcinomas is significantly better than that for squamous cell tracheal cancers (Fig. 1). In this field, survival was slightly better among patients with complete resection than among those with incomplete resection, but the difference was not statistically significant (Fig. 5).

If a complete resection is attempted, the safety of the resection should be the first aim of the surgeon, considering the good long-term results observed in

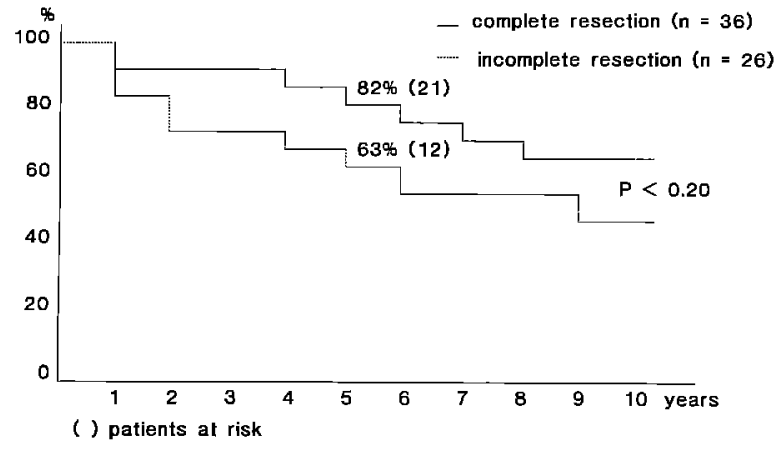

Fig. 5. Actuarial survival according to completeness of resection of adenoid cystic carcinomas.

patients with microscopically positive resection margins, especially with postoperative radiotherapy. ${ }^{1,7}$ In fact, adenoid cystic carcinomas are known for their proclivity to extend for long distances submucosally and perineurally, and the finding of malignant cells at a distance from the gross tumor is common. ${ }^{1}$ The positive lymph node rate in adenoid cystic carcinomas was too low in our series to study the prognostic significance of this factor.

The place of postoperative radiotherapy in the treatment of resectable adenoid cystic carcinomas is extremely difficult to determine; postoperative radiotherapy could be recommended for patients with positive resection margins or nodes. Grillo and Mathisen ${ }^{1}$ recommend such treatment for all patients. As in other series, ${ }^{1,2}$ long-term survival was decreased by the occurrence of distant metastases. In contrast, in patients with tracheal cancers longterm survival was decreased by the occurrence of second primary malignancies generally related to tobacco habits (lung and tongue cancers). Surgical resection remains the treatment of choice for lowgrade malignant tumors (such as carcinoid or mucoepidermoid tumors), but benign tumors are now generally treated conservatively by laser therapy or cryotherapy. ${ }^{12}$

Postoperative course. Morbidity and mortality associated with tracheal resections for tumors remain high, especially in our series. This was due in part to the multicenter nature of this series; in fact, even though the surgical techniques have been well described, ${ }^{1,2,13,14}$ for some surgical teams of this series the experience reported reflects a period of learning about surgical techniques and management. Some teams operated on only a few patients, and only seven teams collected data from 10 patients or more. In the team who collected data from 41 
patients, the mortality rate was $7.5 \%$, which was not very different from those of Grillo and Mathisen ${ }^{1}$ and Pearson and coworkers. ${ }^{2}$ Furthermore, we demonstrated that postoperative mortality was three times higher for tracheal cancers than for adenoid cystic carcinomas or other tumors. As a result, the predominance of tracheal cancers in this series may explain the slightly higher mortality rate. The postoperative mortality rate was $6 \%$ among patients with adenoid cystic carcinoma or other tumors. The high mortality rate associated with tracheal cancers was probably related to the frequency of extensive tumors requiring extended resections and to the predominance of cigarette smokers in this group.

Postoperative complications were significantly correlated with the difficulty of the surgical procedure (length of resection, laryngotracheal or carinal resections). On the other hand, no increase in postoperative complications was noted to be associated with positive resection margins or lymph nodes. Furthermore, the postoperative course directly influenced the long-term functional results, with a high rate of granulomas or stenosis in cases of postoperative locoregional complications. These stenoses were probably caused by partial necrosis of the suture related to excessive anastomotic tension or loss of the blood supply. As in the series of Grillo and Mathisen, ${ }^{1}$ postoperative complications were more commonly associated with carinal resections than with standard tracheal resections. In these complex surgical procedures not only may surgical difficulties be present, some difficulties may occur during the intraoperative ventilation, followed by pulmonary complications.

Surgical procedure. To lessen tension on the anastomosis, adjunctive procedures can be used in addition to systematic pretracheal dissection and cervical flexion. In this series, 63 patients underwent laryngeal release, which seems useful in patients undergoing extensive resection of the midtrachea. Some of these patients had aspiration on deglutition, but most cases resolved with time. For Grillo and Mathisen, ${ }^{1}$ laryngeal release has not generally translated to relaxation after carinal resections, and in such procedures they recommends hilar dissection with a complete incision of the pericardium around the hilum.

Surgical resection is the treatment of choice of tracheal tumors. Postoperative courses remain difficult in extended resections, particularly in cases of carinal or laryngotracheal resection. Such operations should be performed by experienced teams.
Primary relief of the tracheal obstruction by laser therapy should be considered to better prepare the patient for the resection, to decrease the inflammation of the trachea, and to better assess the extent of the tumor and the possibility of a safe and complete resection. Perfect surgical technique is mandatory to avoid anastomotic tension and to preserve the blood supply of the trachea. The place of adjuvant treatment must be determined by further randomized series. Long-term prognosis of tracheal cancers is worsened by the occurence of second primary malignancies, and that of adenoid cystic carcinomas is worsened by the occurence of distant pulmonary metastases.

\section{REFERENCES}

1. Grillo HC, Mathisen DJ. Primary tracheal tumors: treatment and results. Ann Thorac Surg 1990;49:69-77.

2. Pearson FG, Todd TR, Cooper JD. Experience with primary neoplasms of the trachea and carina. J THORAC CARDIOVASC SURG 1984;88:511-8.

3. Gelder CM, Hetzel MR. Primary tracheal tumours: a national survey. Thorax 1993;48:688-92.

4. Eschapasse H. Les tumeurs trachéales primitives: traitement chirurgical. Rev Fr Mal Respir 1974;2:425-46.

5. Houston HG, Payne WS, Harrison EG. Primary cancers of the trachea. Arch Surg 1969;99:132-9.

6. Pearson FG, Cooper JD, Nelems JM, Van Nostrand AW. Primary tracheal anastomosis after resection of the cricoid cartilage with preservation of recurrent laryngeal nerves. J Thorac Cardiovasc Surg 1975;70:806-16.

7. Dedo HH, Fishman NH. Laryngeal release and sleeve resection for tracheal stenosis. Ann Otol 1968;78:285-96.

8. Montgomery WW. Suprahyoïd release for tracheal stenosis. Arch Otolaryngol 1974;99:255-60.

9. Kaplan EL, Meier P. Nonparametric estimation from incomplete observation. J Am Stat Assoc 1958;53:457-81.

10. Rostom AY, Morgan RL. Results of treating primary tumours of the trachea by irradiation. Thorax 1978;33:387-93.

11. Fields JN, Rigaud G, Emami BN. Primary tumors of the trachea: results of radiation therapy. Cancer 1989;63:2429-33.

12. Rusch VW, Schmidt RA. Tracheal schwannoma: management by endoscopic laser resection. Thorax 1994;49:85-6.

13. Grillo HC. Carinal reconstruction. Ann Thorac Surg 1982; 34:356-73.

14. Grillo HC. Tracheal tumors: surgical management. Ann Thorac Surg 1978;26:112-25.

\section{Discussion}

Dr. Claude Deschamps (Rochester, Minn.). I congratulate Regnard and coworkers on an impressive collective series of tracheal resections. I have only three technical questions. What is your standard approach and mode of ventilation for a carinal resection? Do you use vascularized tissue, such as omentum or muscle, to wrap the anastomosis; if so, in what circumstances? Finally, did you ever have to use a prosthesis for an extensive tracheal resection?

Dr. Regnard. In response to your first question, I can 
give you, first of all, the different approaches used in this series for carinal resection. Eight patients were operated on through a median sternotomy, 13 through a right posterolateral thoracotomy, and three through a trapdoor incision. For this collective group of operations, it therefore seems that the best approach for carinal resection was the right posterolateral thoracotomy. Our opinion at Marie Lannelongue Hospital is quite different, however. We consider the best approach for carinal resection to be an anterior approach, generally performed through a full median sternotomy, if necessary combined with cervicotomy. In fact, with an anterior approach you can dissect the entire trachea, mobilize the entire trachea from the larynx to the main bronchus, and if necessary you can perform a laryngeal release or a hilar release; the limits of this approach are probably some difficulties in the dissection of the left main bronchus because of the presence of the aortic arch. Generally, however, we think that the first centimeters of the left main bronchus could be dissected and carinal resection could be performed this way. Furthermore, we consider that intraoperative ventilation is easier in patients in the supine position with an anterior approach, and we generally use jet ventilation for this type of resection. With this technique you can ventilate both lungs with two small catheters without difficulties and without damage.

In response to your second question, we do not use systematically vascularized tissue to cover the anastomosis. I think only patients who have previously received high doses of radiation or high doses of steroids for a prolonged time will benefit from vascularized tissue to cover the anastomosis. In these cases, I think that the best flap is probably an omental flap, which can easily be harvested with an additional small laparotomy. In two other instances, I think that it is better to cover the anastomosis: first, when the anastomosis is just against the innominate artery, in which case we generally use cervical muscles, sternocleidomastoidal muscles, to cover the anastomosis; and second, when the anastomosis is just against the right pulmonary artery after carinal resection, in which case we generally use fat tissue, mediastinal tissue, and pericardium to cover the anastomosis.

In response to your third question, I do not know whether there is a place for prosthetics in tracheal resection. We never use prostheses because published results have not been very encouraging. If a complete resection is not possible because of longitudinal extent of the disease, we prefer to perform laser treatment combined with radiotherapy and chemotherapy rather than using a prosthesis.

Dr. F. Griffith Pearson (Toronto, Ontario, Canada). I congratulate the French Thoracic Surgery Society for putting that information together. I am sure that it was hard work collecting data from so many surgeons and centers. You have produced some very useful and inter. esting information concerning a rare problem with which few centers or surgeons have an opportunity to obtain significant experience.

Dr. Grillo of Boston has more personal experience with this tumor than anyone I know of, and he may choose to comment as well. I wish to emphasize some of your points regarding adenoid cystic carcinoma. Your data clearly show that this is a slowly progressive tumor and that the time from resection to local recurrence is frequently prolonged-almost 5 years in cases that did recur. I recently had a patient who died of a local recurrence that only became evident 27 years after the original incomplete resection. You also note that there are few metastases in lymph nodes in adenoid cystic cancers; most of the nodal metastases are probably due to direct overgrowth by the primary tumor itself. Some of these patients have pulmonary metastases, which may also remain asymptomatic for extremely long periods, and I would appreciate your comments in this regard. In my opinion, such asymptomatic metastases are not necessarily a contraindication to resection of the primary tumor in selected cases.

Finally, you make a plea for radiotherapy because of the narrow margins that are possible after resection of the mediastinal trachea. I support your recommendation. To my knowledge, adenoid cystic cancer is highly radioresponsive in many cases. With radiotherapy alone, however, no one has reportedly survived more than 10 years. From personal communication, I understand that Dr. Grillo has seen one patient who survived as long as 11 years after irradiation only. In our own experience (I believe we have operated on about 36 patients with adenoid cystic cancer in a 30 -year period), I have not seen a local recurrence in any patient in whom a complete resection was achieved at the initial operation. After an incomplete resection, however, the time to recurrence averaged about was 8 years.

I greatly enjoyed the report, and I again congratulate you on what must have been very hard work digging out that information.

Dr. Regnard. I totally agree with your comments.

\section{Appendix}

French Society Thoracic and Cardiovascular Surgery, authors and centers participating in the study: J. Bellamy, MD (Saint-Cloud); P. Bergeron, MD, P. Rudondy, MD (Marseilles), A. Bisson, MD (Suresnes); J. Borrelly, MD (Nancy); G. Chauvin, MD, B. Pena, MD (Avignon); L. Couraud, MD, J. Jougon, MD (Bordeaux); M. Dahan, MD, J. Berjaud, MD (Toulouse); B. Debesse, MD, M. Riquet, MD (Paris); C. Evrard, MD, B. Martel, MD (Caen); J. M. Faillon, MD (Lille); J. P. Gamondes, MD (Lyon); B. Guibert, MD (Lyons-Sud); L. K. Lacquet, MD (Nijmegen); G. Maggi, MD, A. Cavallo, MD (Turin); C. Marty-Anne, MD, H. Mary, MD (Montpellier); J. L. Michaud, MD, O. Baron, MD (Nantes); P. Mondine, MD, J. A. Barrat, MD (Brest); G. Morand, MD, G. Massard, MD (Strasbourg); J. F. Regnard, MD, P. Levasseur, MD (Le Plessis Robinson); H. Richelme, MD, J. Mouroux, MD (Nice); R. Sarrazin, MD, P. Y. Brichon, MD (Grenoble); P. Schneider, MD, I. Vogt-Moykopf, MD (Heidelberg); J. Sulzer, MD (Le Mans); B. Toumieux, MD, C. Dromer, MD (Tours); H. Viard, MD, A. Bernard, MD (Dijon); and A. Wurtz, MD, L. Gambiez, MD (Lille). 